\title{
Una introducción a los espacios vectoriales
}

\author{
y al lenguaje logo \\ a través del ordenador
}

\section{Antonio Bautista García-Vera *}

Universidad Complutense

\section{INTRODUCCION}

Son varias las razones que nos han llevado a escribir este artículo. La primera de ellas se centra en el papel importante que tiene la geometría en la formación y desarrollo del individuo porque debido a su claro soporte intuitivo facilita el desarrollo de la imaginación, aumenta la sensibilidad y la capacidad creadora. En otro sentido, el estudio de las propiedades geométricas provoca en el alumno la necesidad de rigor en el lenguaje, y la conveniencia de dudar de sus percepciones y de relativizar los juicios que se apoyan en ellas, a la vez que estimula la búsqueda de verdades que están por encima de nuestra propia sensibilidad.

En esta línea Sherard III (1981) apunta un grupo de razones por las que considera que la Geometría es una habilidad básica. Entre otras, indica que proporciona oportunidades para desarrollar la percepción espacial y además porque nos sirve de vehículo para estimular y ejercitar destrezas generales de pensamiento y habilidades para resolver problemas.

Una segunda razón la indicó la UNES-
CO en 1972 al apuntar la falta de criterios para lograr un consenso en el desarrollo curricular de la Geometría:

"La concepción moderna de la enseñanza de la Geometría sigue sujeta a nuevas investigaciones pedagógicas y el establecimiento de un programa aceptable para ella es actualmente uno de los problemas curriculares más difíciles."

Trece años después la situación sigue siendo parecida porque la Geometría tiene que ser enseñada a alumnos de edades comprendidas entre los doce y diecisiete años, con aptitudes y capacidades de comprensión y razonamiento diferentes.

Una tercera razón se debe a la situación crítica que atraviesan los métodos de enseñanza de la Geometría en la actualidad. Landa (1978), Choquet (1983), Santaló (1980) y De Lorenzo (1980) entre otros, señalan que el maestro normalmente sólo se ocupa de ofrecer a los alumnos conocimientos sobre contenidos geométricos y se olvida de darles los medios para operar con dichos contenidos, de enseñarle cómo indagar, reflexionar y pensar a través de la asimilación y de la aplicación de esos conocimientos.

" Dirección del autor: Universidad Complutense, Facultad de Filosofía y C.C. de la Educacion. Departamento de Metodología Educativa (Didáctica). 28040 Madrid. 
Choquet en este setido apunta uno de los principios fundamentales para la enseñanza de la Geometría, el que debe basarse en la observación, y que los conceptos geométricos deben elaborarlos los alumnos a partir de la experiencia.

Aparte de estas limitaciones desveladas desde el análisis de las estructuras psicológicas y lógicas de la actividad mental existentes durante el aprendizaje de la Geometría, desde una perspectiva externa, referida a los métodos y medios de enseñanza de esta disciplina, surgen otras deficiencias que explican tal marginación. Nos referimos a la falta de herramientas que ejecuten con exactitud y rapidez los planes de representación y creación de formas geométricas elaborados en la mente del alumno.

Otro grupo de razones surgen desde una perspectiva computacional.

La actual «Revolución Informática» afecta a la adaptación de los estudiantes de hoy a la cercana sociedad interconectada, así como a la integración de éstos al mundo laboral informatizado en que les tocará trabajar.

Ante este planteamiento, la llegada de los microordenadores a los centros escolares tienen los siguientes grupos de objetivos:

- Lograr una alfabetización informática (Computer Literacy) en los alumnos. Entendida, siguiendo a Watt (1980), Klassen (1981) y Molnar (1981), como el conjunto mínimo de conocimientos, capacidades y destrezas, sobre equipos y programas de ordenador, que permita a un individuo, "habérselas eficazmente con el mundo".

- Mejorar los procesos de enseñan$\mathrm{za}$ aprendizaje, de las materias del currículo, que se desenvuelven en el aula.

Hay dos posibilidades de programar el primer grupo de objetivos en el desarrollo del currículo:

- De forma independiente, por medio de una asignatura con contenidos propiamente informáticos.

- Conjuntamente con un área del segundo grupo.
Dentro de la segunda posibilidad es evidente que cualquier forma de utilización del microordenador no permite aprender unos contenidos auxiliares sobre informática (en nuestro caso, sobre el lenguaje de programación LOGO) a la vez que unos contenidos básicos de cualquier área (en nuestro caso de matemáticas) ${ }^{1}$.

La breve historia de la introducción del ordenador en el campo educativo apunta dos grandes modos de utilización: Como director del aprendizaje de los alumnos y como medio del mismo.

El primero, que llamaremos "clásico», se refiere a la enseñanza asistida por ordenador o CAI (Computer Assisted Instruction). En él el ordenador es el responsable de presentar progresivamenie a los alumnos los contenidos objeto de aprendizaje. El orden de presentación depende de las respuestas que vayan dando los estudiantes.

Puede apreciarse en esta concepción metodológica que el ordenador es un sustituto de un libro de enseñanza programada y el alumno un ser programado, cuya actividad se limita a dar respuesta a las cuestiones que le formula el ordenador.

Otro inconveniente que presenta este tipo de utilización se refiere al «courseware" ${ }^{2}$. En este sentido, y en la medida en que el ordenador necesita de unos programas (referidos a unos contenidos concretos) para dirigir los procesos de aprendizaje y valorar los conocimientos adquiridos por el alumno, el profesor tiene dos alternativas; o preparar él mismo el software educativo o adquirirlo en el mercado. Ambas presentan dificultades, porque no siempre el profesor está capacitado para elaborarlo y porque, además de la baja calidad que frecuentemente se observa en los programas existentes, no siempre dispone el mercado de courseware para esas materias específicas y para poder ser utilizado por una determinada marca de microordenador.

Este tipo de utilización se ha venido desarrollando desde las experiencias pioneras de Suppes, Loftus y Jerman (1969); Suppes (1972), etc., hasta los actuales lenguajes y sistemas de autor.

La segunda forma metodológica de introducir el ordenador en el aula se centra en la filosofía propuesta por Papert, S. (1980), seguida por Reggini, H. (1982) y 
defendida actualmente por Solomon, C. (1982),; Watt, D. (1984),; Lawler, B. (1984); Howe, J. AM. y otros (1984) y Billstein, R. (1985), entre otros.

Consideran estos autores que el microordenador debe ser un medio que se pone a disposición del alumno para que: explore problemas, almacene y recupere datos, compruebe soluciones, simule procesos, realice cálculos y aborde cuestiones usando varias estrategias.

En esta segunda utilización metodológica, el alumno es un ser activo que investiga y descubre las soluciones de unas situaciones problemáticas planteadas por el profesor, y el microordenador una máquina que es programada por el estudiante.

Ante estos dos tipos de utilización es evidente que sólo el segundo permite a los alumnos programar el ordenador para desarrollar algún proyecto, cálculo, o ejecutar cualquier plan de resolución de alguna situación problemática que les haya sido propuesta. Para poder llegar a programarlo necesitan conocer la estructura y órdenes de algún lenguaje de ordenador.

Esta necesidad, además de ser un elemento motivante para el aprendizaje, justifica la segunda alternativa de planificación de contenidos apuntada al principio de este artículo: que los contenidos informáticos estén integrados en la misma programación de las materias de un área del currículo escolar. Según Dijkstra (1971) y L. F. Paradela (1985) en una revisión de las recomendaciones del proyecto Atenea, uno de los lenguajes de programación más convenientes, por su estructuración, para la enseñanza es LOGO.

Estas razones y argumentos nos llevan a exponer en este artículo cómo los alumnos de 2. de BUP pueden aprender con el ordenador unos conceptos sobre espacios vectoriales utilizando el lenguaje de programación LOGO.

\section{BASES PSICOLOGICAS Y PEDAGOGICAS DE NUESTRA PROPUESTA METODOLOGICA}

¿Qué estrategia de intervención soslayará las dificultades que inciden en los procesos de enseñanza/aprendizaje de los conceptos de espacios vectoriales e integrará a la vez la adquisición de contenidos sobre el lenguaje de ordenador: LOGO?

Tiene que ser una metodología basada en un modelo instructivo que fundamente científicamente la enseñanza y permita analizar la experiencia educativa. Para soslayar las dificultades que han llevado a la Geometría a su estado de marginación, es necesario que este modelo tenga entre sus componentes principales los aspectos psicológicos de aprendizaje y desarrollo del alumno.

Un modelo instructivo que integra coherentemente estos aspectos es el propuesto por Gimeno Sacristán (1985). Según este enfoque toda estrategia de enseñan$\mathrm{za}$ /aprendizaje surgirá de las opciones que realice todo didacta en torno a las siguientes cuatro dimensiones o referentes:

\section{A: OBJETIVOS}

B: SUBSISTEMA DE ENSEÑANZA. Que consta de las subdimensiones: Contenidos, relaciones de comunicación, medios, organización y evaluación.

\section{C: SUBSISTEMA PSICOLOGICO.}

En el que se tiene presente la interacción desarrollo-aprendizaje.

D: REFERENTE SOCIOCULTURAL.

Frente a cada uno de estos componentes, exceptuando el referente sociocultural que no tiene unas implicaciones directas, vamos a ir especificando las posturas y opciones que eviten las dificultades apuntadas, para después formalizar frente a ellas nuestra propuesta metodológica.

\section{A. Objetivos}

Es la primera dimensión de este modelo. Hay que entenderla no sólo como una serie de estados a los que hay que llegar, sino también como un conjunto de referencias orientativas sobre el camino a seguir.

Dentro de esta dimensión vamos a explicitar los objerivos que nos proponemos, tanto sobre espacios vectoriales como so- 
bre LOGO, porque este hecho facilitará posteriormente la elección de: medios, tipo de comunicación y, en suma, la concrección de la metodología o estrategia de acción.

De forma explícita, los objetivos de ambas áreas de conocimiento son: a) Sobre el lenguaje $L O G O$ de programación:

Al final del proceso instructivo el alumno será capaz de:

$a_{1}$ ) Comprender y aplicar correctamente en un programa las siguientes órdenes ${ }^{3}$ :

\begin{tabular}{|c|c|c|}
\hline $\begin{array}{l}\text { Ordenes } \\
\text { a la tortuga }\end{array}$ & Abreviatura & $\begin{array}{l}\text { Traducción al castellano } \\
\text { y observaciones }\end{array}$ \\
\hline PRINT & PR & IMPRIME (lo que viene a continuación) \\
\hline FORDWARD & $\mathrm{FD}$ & AVANZA (un número $\mathrm{N}$ de pasos) \\
\hline $\mathrm{BACK}$ & $\mathrm{BK}$ & RETROCEDE (un número $\mathrm{N}$ de pasos) \\
\hline RIGHT & RT & $\begin{array}{l}\text { GIRA A LA DERECHA (un número } \mathrm{N} \text { de } \\
\text { grados) }\end{array}$ \\
\hline LEFT & LT & $\begin{array}{l}\text { GIRA A LA IZQUIERDA (un número } \mathrm{N} \text { de } \\
\text { grados) }\end{array}$ \\
\hline PENUP & PU & $\begin{array}{l}\text { LEVANTA LA PLUMA (la tortuga no deja } \\
\text { rastro) }\end{array}$ \\
\hline PENDOWN & PD & BAJA LA PLUMA (la tortuga deja rastro) \\
\hline SENTENCE & SE & UNE varias palabras (listas o entradas) \\
\hline REPEAT & & $\begin{array}{l}\text { REPITE (repite } \mathrm{N} \text { numero de veces lo que se } \\
\text { ponga entre corchetes) }\end{array}$ \\
\hline SETPOS & & $\begin{array}{l}\text { VETE A (la tortuga se mueve hace las coor- } \\
\text { denadas de un punto de la pantalla que se dan } \\
\text { como entrada) }\end{array}$ \\
\hline SETHEADING TOWARDS & & $\begin{array}{l}\text { MIRA HACIA (el triángulo que representa a } \\
\text { la tortuga gira hacia las coordenadas de un pun- } \\
\text { to que se dan como entrada) }\end{array}$ \\
\hline HOME & & $\begin{array}{l}\text { A CASA (la tortuga vuelve al centro de la } \\
\text { pantalla apuntando hacia arriba) }\end{array}$ \\
\hline $\mathrm{XCOR}$ & & $\begin{array}{l}\text { COORDENADA X (de la posición actual de } \\
\text { la tortuga) }\end{array}$ \\
\hline YCOR & & $\begin{array}{l}\text { COORDENADA Y (de la posición actual de } \\
\text { la tortuga) }\end{array}$ \\
\hline
\end{tabular}

$a_{2}$ ) Construir y utilizar con síntaxis correcta, procedimientos que contengan parámetros de entrada y salida.

$a_{3}$ ) Especificar y utilizar correctamente en programas las siguientes operaciones y funciones LOGO:

\begin{tabular}{cl}
$\begin{array}{c}\text { SIMBOLO } \\
\text { OPERADOR LOGO }\end{array}$ & \multicolumn{1}{c}{ SIGNIFICADO } \\
\hline+ & Suma \\
- & Resta \\
$\%$ & Multiplicación \\
$/$ & División \\
SQRT & Raíz cuadrada
\end{tabular}

\section{b) Sobre espacios vectoriales:}

Al final del proceso de instrucción el alumno será capaz de:

$b_{1}$ : Dados dos puntos $A=\left(X_{1}, Y_{1}\right)$, $\mathrm{B}=\left(\mathrm{X}_{2}, \mathrm{Y}_{2}\right)$ representar el vector $\overrightarrow{\mathrm{AB}}$.

$\mathrm{b}_{2}$ : Descubrir si son equipolentes dos vectores. polencia es una relación de equivalencia.

$b_{5}$ : Dado un vector $\overrightarrow{\mathrm{AB}}$ representar un vector perteneciente a esa clase que tenga su origen en el punto $\mathrm{C}=\left(\mathrm{X}_{1}, \mathrm{Y}_{1}\right)$.

$b_{6}$ : Calcular y representar la suma de dos vectores libres.

$b_{7}$ : Definir correctamente las propiedades conmutativa, asociativa, elemento neutro y simétrico de la suma de dos vectores libres.

$b_{8}$ : Calcular y representar la diferencia de dos vectores libres.

$b_{9}$ : Representar el resultado de la multiplicación de un vector libre por un número real.

$b_{1} \mathrm{o}$ : Definir correctamente las propieda$\operatorname{des}(a+b)=r a+r b ;(r+s) \cdot a=$ $=\mathrm{ra}+\mathrm{sa} ;(\mathrm{rs}) \mathrm{a}=(\mathrm{sa}) ; 1 \mathrm{a}=\mathrm{a}$. Siendo $a, b$ vectores y $r, s$ números reales.

$b_{11}$ : Dados dos vectores libres $a_{1}$ y $a_{2}$, sabrá dibujar un representante de la combinación lineal $2 a_{1}-1 / 2 a_{2}$. 
B) Subsistema de enseñanza. Dentro de esta segunda dimensión vamos a ir tomando decisiones respecto a los siguientes subsistemas:

$\mathrm{B}_{1}$ : Contenidos. En los objetivos de enseñanza propuestos anteriormente están implícitos unos contenidos científicos (órdenes LOGO y conceptos sobre espacios vectoriales) y las funciones psicológicas de comprensión y aplicación según Bloom. En cuanto estos objetivos son básicos para la comprensión y aplicación de conocimientos más complejos, van dirigidos a todos los alumnos de $2 .^{\circ}$ de BUP. La secuencialización que hemos hecho de los contenidos ha sido atendiendo al orden creciente de complejidad.

$\mathrm{B}_{2}$ : Relaciones de comunicación. La estructura formal de esta relación será la de una multiplicidad de emisores y receptores. De esta forma evitaremos una de las dificultades señaladas que normalmente tienen lugar en la enseñanza de los espacios vectoriales, como es la ausencia de medios y la falta de elaboración de conceptos por los alumnos partiendo de la observación y basándose en la experiencia. Esta dificultad la soslayamos utilizando como emisores en nuestra estrategia metodológica, además de al profesor, a un medio especializado que permita representar y operar con vectores, y ejecutar con precisión y rapidez las estrategias o planes de resolución de algunas situaciones problemáticas propuestas. Este medio es el ordenador.

Las relaciones de comunicación que consten en la estrategia deben ser flexibles, es decir, que permitan entre los elementos del proceso de enseñanza/aprendizaje la alternancia de roles. Asi, un emisor puede convertirse en receptor en un momento dado y viceversa. De esta forma, el alumno en algunos momentos será receptor pero en otros casos se convertirá en emisor y podrá descubrir mediante la investigación y la indagación. Eliminamos así otra de las dificultades que operan en metodologías de enseñanza de los espacios vectoriales.

Desde un punto de vista de los contenidos de la comunicación, además de aquellos que tienden a proporcionar informaciones y estructurar la situación de enseñanza/aprendizaje, tendremos presente en nuestra metodología los que Gimeno Sacristán llama de "control de conducta». A través de éstos, el profesor comprobará el nivel de comprensión adquirido por los alumnos y éstos, a su vez, podrán verificar hipótesis, indagar la bondad de un plan o estrategia para resolver una situación problemática, etc.

$\mathrm{B}_{3}$ : Medios. Son varias las razones que nos han llevado a utilizar en nuestra propuesta metodológica el ordenador. La primera de ellas se debe a que entre los objetivos que hemos propuesto figuran órdenes del lenguaje LOGO. Es evidente que para comprender y aplicar esas órdenes es necesario un ordenador.

Otra de las razones se debe a que es un medio que permite representar y visualizar fácilmente en la pantalla operaciones con vectores. En este sentido, el ordenador hace más accesibles intuitivamente a los alumnos determinados contenidos abstractos como son los referidos a espacios vectoriales. Volveremos a tocar este punto cuando tratemos el subsistema psicológico.

Ya apuntábamos en líneas anteriores la forma de utilizar el ordenador, al que consideraremos como un medio del que los alumnos se sirven para investigar o ejecutar con precisión y rapidez planes de resolución de situaciones problemáticas. Esta opción tiende a soslayar la pasividad que mantenía el alumno en la metodología tradicional y que, como apuntábamos, ocasionaba dificultades en el aprendizaje de conceptos sobre espacios vectoriales.

$\mathrm{B}_{4}$ : Organización. Gimeno Sacristán apunta que la organización «tiene que ser un elemento facilitador de las opciones que se hayan tomado en los demás elementos del modelo» (4). En esta subdimensión tenemos que organizar las opciones que hemos ido tomando para facilitar la estrategia didáctica.

Pero el aprendizaje necesita una serie de condiciones materiales y psicológicas, condiciones que a la vez supondrán cierta normatividad que habrá que respetar en la organización de la enseñanza. Por esta razón las opciones a concretar frente a este elemento organizativo las tomaremos después de revisar el subsistema psicológico.

Según Gimeno la quinta subdimensión del subsistema de enseñanza, la evaluación, no es un elemento respecto del cual haya que decidir para establecer una determinada estrategia de enseñanza. Por esto no la tendremos presente en nuestra propuesta metodológica. 
C) Subsistema psicológico. Tiene como fin fundamentar y garantizar que la propuesta metodológica que proponemos lleve a los alumnos a unos resultados de aprendizaje deseados. A su vez, va a proporcionar una explicación de los procesos mentales desarrollados durante la acción pedagógica.

Ante la variedad de teorías y modelos de aprendizaje necesitamos como referente uno que contemple y ponga de relieve los procesos ocurridos en la mente de quien aprende. Uno de éstos, que integra las orientaciones de Gagné y las aportaciones de las teorías de procesamiento de la información, es el propuesto por Pérez Gómez (1981). Hace referencia este modelo a tres dimensiones:

- Las condiciones del aprendizaje.

- Los procesos del aprendizaje.

- Los resultados del aprendizaje.

Como las condiciones y resultados del aprendizaje pretendido por nuestra propuesta metodológica han quedado en parte concretados en las opciones tomadas al revisar el subsistema de enseñanza, nos centraremos en los procesos.

Considera Pérez Gómez ${ }^{5}$ que durante el aprendizaje tienen lugar en la mente los siguientes procesos:
$\mathrm{C}_{1}$ : atención y selección.
$\mathrm{C}_{2}$ : codificación y asimilación
$\mathrm{C}_{3}$ : almacenamiento organizado.
$\mathrm{C}_{4}$ : recuperación constructiva.
$\mathrm{C}_{5}$ : utilización y transferencia.

$\mathrm{C}_{1}$ : Atención y selección. Hemos señalado que una de las dificultades que se han puesto de manifiesto en la enseñanza de la geometría en general y de los espacios vectoriales en particular se refiere a la falta de experiencias y condiciones del exterior que sean adecuadas a los alumnos a los que iban dirigidos esos contenidos.

Esta carencia, a su vez, va a dificultar la puesta en marcha de los procesos de atención y selección, pues pocas cosas son más contraproducentes para un alumno desde un punto de vista del aprendizaje, que la necesidad de dominar conceptos y contenidos que no tengan sentido para él o que sean extraños y ajenos a su mentalidad.

En nuestra propuesta metodológica vamos a soslayar esta dificultad de dos formas. Una de ellas, siguiendo a Butts (1980), Jacobson, Lester y Stenger (1980) y Osborne y Kasten (1980) entre otros, es suscitar esos procesos de atención y selección a través de la presentación de situaciones problemáticas ${ }^{6}$ en las que estén incluidos los contenidos objeto de aprendizaje. Situaciones de aprendizaje que estarán relacionadas con los intereses y necesidades de los alumnos a los que van dirigidas.

Una segunda forma de elicitar esos procesos la proporciona el propio ordenador. Según Malone (1981) y Malone (1981b), es un medio que por sí mismo es motivante y atrayente para los estudiantes. Utilizándolo según hemos comentado en líneas anteriores, el ordenador es capaz de retar y despertar la fantasía e interés de los alumnos.

$\mathrm{C}_{2}$ : Codificación y asimilación. El proceso de codificación conlleva codificar simbólicamente un estímulo de acuerdo con varios factores (características físicas, semánticas, etc.).

Este proceso será amplificado y ampliado si el alumno tiene que representar los elementos del problema y proyectar sus planes de resolución en un lenguaje de ordenador o sistema de codificación adicional que ponemos a disposición del alumno.

Desde el punto de vista de los procesos de asimilación de los contenidos implícitos en las situaciones problemáticas propuestas a los alumnos, según Hayes (1978), asimilaremos mejor un problema cuando nos hayamos hecho una representación interna de él. ¿Y si hacemos dos representaciones de la situación problemática porque dispongamos de otro modelo de codificación/representación a través de un lenguaje de ordenador? Según Hayes, tendremos aún más probabilidad de asimilarlo y comprenderlo porque un problema puede representarse de muchas formas y cuantas más realice un alumno más posibilidades de integración tiene en sus esquemas cognitivos existentes y con más posibilidades contará para ejercitar la actividad asimiladora de estos esquemas.

La posibilidad de elicitar estos procesos en los estudiantes que se abre con nuestra propuesta metodológica, favorecerá en éstos la elaboración de conceptos sobre espacios vectoriales, partiendo de experiencias de enseñanza.

$\mathrm{C}_{3}$ : Almacenamiento organizado. Según 
Pérez Gómez, sólo la información que está organizada jerárquicamente, puede retenerse durante largos períodos de tiempo y puede ser utilizada en programas de solución de problemas.

Estos procesos de almacenamiento organizado se verán favorecidos si los alumnos disponen de un ordenador en el que pueden almacenar (siempre ordenadamente a través de ficheros) gran número de datos en los soportes permanentes de información (disquetes, cassettes, etc.). Posteriormente, el ordenador en cualquier momento puede ofrecer la información organizada en la pantalla.

Vuelve a ser aqui el ordenador un buen medio que favorece los procesos de almacenamiento organizado, porque antes de que el alumno la grabe necesita tenerla almacenada y organizada en su mente.

$\mathrm{C}_{4}$ : Recuperación constructiva. Conllevan estos procesos mentales la subsiguiente utilización de la información almacenada como guía de la actuación.

Al utilizar unas situaciones problemáticas como punto de partida en nuestra propuesta metodológica, los alumnos crearán para resolverlas unos planes o estrategias. Para ello irán recuperando en esta fase las informaciones de forma coherente con la meta o metas que especifique el enunciado del problema.

$\mathrm{C}_{5}$ : Utilización $y$ transferencia. Estos procesos son importantes para lograr la función psicológica de aplicación de los objetivos sobre espacios vectoriales y LOGO que nos hemos propuesto.

Estos procesos serán generados en dos fases diferentes de la metodología que vamos a proponer. En la primera, el alumno aborda la comprensión del enunciado de la situación problemática, y en ella aparecen ya utilizados los conceptos objeto de aprendizaje. En la segunda, el alumno comprende la representación y planificación de estrategias de resolución, donde según Abelson y diSessa (1984), al operar con vectores utilizando el lenguaje LOGO el alumno dispondrá de dos representaciones diferentes de una misma operación (geometría de la tortuga y geometría de vectores). Al compararlas y trasladar las descripciones de una representación sobre la otra se suscitan procesos estimulares y transferencias que no aparecerían si sólo hubiésemos utilizado una sola.
Como resultado de esta revisión vamos a organizar estas opciones en torno a las fases de resolución de problemas propuestos por los clásicos Dewey (1933) y Polya (1945) y actualmente por Torgerson (1984), Catheart (1984) y Dromey (1982).

Apuntamos una metodología de enseñanza/aprendizaje que permite exponer los contenidos sobre espacios vectoriales y aplicar estrategias de resolución utilizando los conceptos sobre el lenguaje de programación LOGO, que previa y progresivamente han aprendido los alumnos'.

Este planteamiento metodológico debe entenderse como una manera de organizar la situación de aprendizaje, en la que el centro de comunicación es un problema a resolver por parte de los alumnos. Los contenidos del curriculo, objetos de aprendizaje, estarán incluidos bien en el enunciado del problema, bien en el proceso de resolución, bien en los resultados obtenidos. Sus fases son:

1. Planteamiento de una situación problemática relacionada con el mundo real del alumno. Este no conocerá el procedimiento de resolución pero sí tendrá en su memoria los conceptos y operaciones necesarias.

2. Comprensión del problema. Primero en una discusión grupal y después de forma individual, manifestará oralmente las condiciones iniciales $y$ finales del problema.

3. Elaboración de un plan o estrategia de resolución. Primero individualmente por escrito (futuro algoritmo) y en un segundo momento en grupos pequeños (para contrastar con otras planificaciones).

4. ${ }^{\circ}$ Realización del plan. Se hará de forma individual. El alumno dispone del microordenador y del "ambiente» o «micromundo" de aprendizaje LOGO para ejecutar con precisión y rapidez la estrategia elaborada en la fase anterior.

5. Contraste, comprobación y manifestación de los resultados obtenidos. Si éstos no satisfacen las condiciones del problema se vuelve a la fase dos. La importancia de esta fase se debe a que, independientemente de que sean o no correctos los resultados ${ }^{8}$, supone un refuerzo de aprendizaje. Técnicamente, la comprobación se realiza a través de la compilación 0 interpretación ${ }^{9}$ del programa introduci- 
do por el alumno al microordenador. Los resultados de la ejecución del mismo, reflejados en la pantalla del monitor o en el listado de la impresora, permitirán al alumno hacer la verificación.

\section{DESARROLLO DE LOS OBJETI- VOS ${ }^{10}$}

\section{Objetivo}

Para resolver los problemas que les permitan conseguir el objetivo $b_{1}$ utilizando el microordenador, los alumnos deben antes saber definir procedimientos y conocer las siguientes órdenes del lenguaje LOGO:

PU, SETPOS, SE, SETHEANDING TOWARDS, PD, HOME, LT, FD, BK Y RT.

Para representar vectores con microordenador, utilizando LOGO, los estudiantes pueden seguir, entre otras, las siguientes estrategias:

TO REPRE-VECTORES $: \mathrm{N}: \mathrm{X}_{1}: \mathrm{Y}_{1}$ $: \mathrm{X}_{2}: \mathrm{Y}_{2}$

PU

SEPTOS SE $\left(: \mathrm{X}_{1}\right)\left(: \mathrm{Y}_{1}\right)$

SETHEADING TOWARDS SE $\left(: \mathrm{X}_{2}\right)$ $\left(: \mathrm{Y}_{2}\right)$

$\mathrm{PD}$

SETPOS SE $\left(: \mathrm{X}_{2}\right)\left(: \mathrm{Y}_{2}\right)$

FLECHA
PU

HOME

END

Siendo FLECHA el nombre de un procedimiento que pinta un pequeño ángulo agudo, en el extremo del trazo dibujado, para indicar el sentido del vector:

\section{TO FLECHA}

LT 150

FD 5

BK 5

RT 300

FD 5

LT 150

END ${ }^{11}$

Después de dibujar la flecha que tiene un ángulo interno de $30^{\circ}$ y un lado de 5 pasos, deja la torruga en la orientación inicial como se aprecia en la fig. 1.

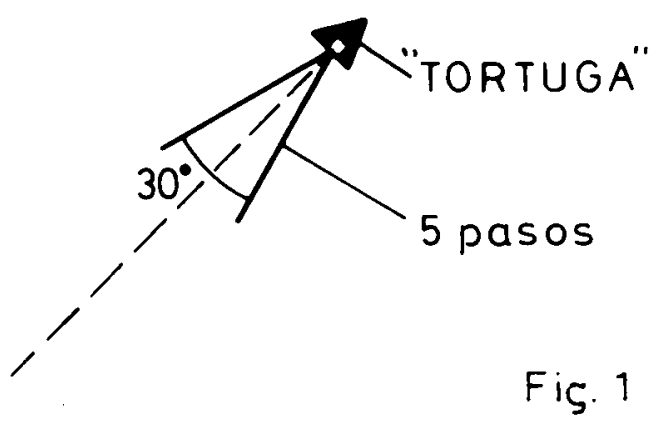

Si previamente quisieran trazar unos ejes cartesianos de referencia, para poder situar el vector representado, lo pueden hacer siguiendo las referencias que indican las coordinadas de la fig. 2

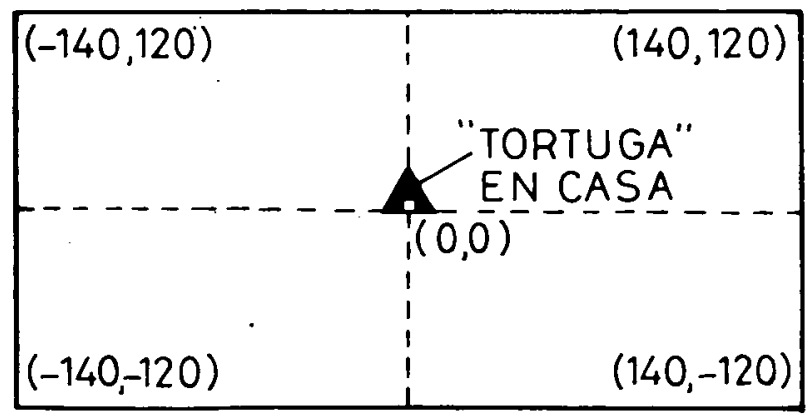

Coordenadas de

la pantalla del

APPLE II 
to:

y utilizando el siguiente procedimien-

TO EJES-CARTESIANOS

ORDENADAS

ABSCISAS

END

Este procedimiento llama a su vez a ORDENADAS y ABSCISAS.

\section{TO ORDENADAS Procedimiento para dibujar REPEAT 24 [FD 10 LT 90 FD 3 BK 3 RT 90] $\}$ el eje deordenadas marcando END intervalos cada 10/pasos.}

TO ABSCISAS

RT 90

REPEAT 28 [FD 10 LT 90 FD 3 BK 3 RT 90]

LT 90

END

\section{Objetivo $b_{2}$}

Los estudiantes sabrán definir procedimientos con parámetros de salida (utilizando OUTPUT) y las órdenes de cálculo $+\mathrm{y} \%$.

Siguiendo la metodología propuesta anteriormente, los alumnos que se enfrenten a un problema centrado en el objetivo de aprendizaje ${ }^{12}$ podrán resolverlo creando entre otros el procedimiento EQUIPOLENTE ${ }^{13}$.

TO EQUIPOLENTE $: N_{1}: X_{1}: Y_{1}: X_{4}$ $: \mathrm{Y}_{4}$

\section{PU}

SETPOS SE $\left(: \mathrm{X}_{1}\right)\left(: \mathrm{Y}_{1}\right)$

SETHEADING TOWARDS SE (MEDIA : $\left.\mathrm{X}_{1}: \mathrm{X}_{4}\right)\left(\right.$ MEDIA $\left.: \mathrm{Y}_{1}: \mathrm{Y}_{4}\right)$

PD

SETPOS SE (MEDIA : $\left.\mathrm{X}_{1}: \mathrm{X}_{4}\right)(\mathrm{ME}-$ DIA : $\left.Y_{1}: Y_{4}\right)$

PU

HOME

END

Siendo MEDIA:

TO MEDIA :X :Y

OUTPUT $(: X+: Y) / 2$

END

Igual hará para dibujar el segundo punto medio:

TO EQUIPOLENTE $: \mathrm{N}_{2}: \mathrm{X}_{3}: \mathrm{Y}_{3}: \mathrm{Y}_{2}$ $: Y_{2}$
EQUIPOLENTE 1., dibujará la distancia media entre el punto de partida del primer vector $\left(X_{1}, Y_{1}\right)$ y el punto final del segundo $\left(\mathrm{X}_{4}, \mathrm{Y}_{4}\right)$.

EQUIPOLENTE 2., trazará la distancia media entre el punto de partida del segundo vector $\left(\mathrm{X}_{3}, \mathrm{Y}_{3}\right)$ y el punto final del primero.

Si ambos trazos coinciden en un punto esos vectores serán equipolentes, pero no lo serán si ambos segmentos se cruzan o no se juntan.

\section{Objetivo $b_{3}$}

Los alumnos deberán haber ampliado, respecto a los conceptos mínimos necesarios para conseguir el objetivo anterior, el conocimiento de las siguientes órdenes LOGO:

PRINT (para agrupar varias entradas), SORT (raiz cuadrada) - (diferencia) y * (producto). Podrán utilizar entre otras las siguientes estrategias de construcción de procedimientos:

TO MODULO-VECTOR :N : $\mathrm{X}_{1}: \mathrm{Y}_{1}$ $: \mathrm{X}_{2}: \mathrm{Y}_{2}$

(PRINT [MODULO DEL VECTOR] $: \mathrm{N}[=]$ CALCU-MODULO $: \mathrm{N}: \mathrm{X}_{1}: \mathrm{Y}_{1}$ $: \mathrm{X}_{2}: \mathrm{Y}_{2}$ )

END

Siendo calcu-módulo el término que llama al procedimiento

TO CALCU-MODULO :N : $\mathrm{X}_{1}: \mathrm{Y}_{1}$ $: \mathrm{X}_{2}: \mathrm{Y}_{2}$ 
OUTPUT SQRT SUMA (RESTA-

CUA : $\mathrm{X}_{1}: \mathrm{X}_{2}$ ) (RESTA-CUA $\left.: \mathrm{Y}_{1}: \mathrm{Y}_{2}\right)$ END

Donde resta-cua es:

TO RESTA-CUA :NUM :NUM $_{2}$ OUTPUT CUADRADO (DIFERENCIA :NUM $:_{1} \mathrm{NUM}_{2}$ )

\section{END}

TO SUMA :NUM $:_{1}$ NUM $_{2}$ OUTPUT : $\mathrm{NUM}_{2}+\mathrm{NUM}_{2}$ END

TO DIFERENCIA :NUM :NUM $_{2}$ OUTPUT :NUM N $_{2}$ NUM $_{1}$

END

TO CUADRADO :X

OUTPUT : $\mathrm{X} *: \mathrm{X}$

END

Ya comentamos las posibilidades de verificación gráfica que tiene el microordenador. A continuación presentamos un ejemplo de cómo aplicarlas en la adquisición de este objetivo.

TO VERIFICACION-VALOR-MODULO :MOD :N : $\mathrm{X}_{1}: \mathrm{Y}_{1}: \mathrm{X}_{2}: \mathrm{Y}_{2}$

PU

SETPOS SE $\left(: \mathrm{X}_{1}\right)\left(: \mathrm{Y}_{1}\right)$

SETHEADING TOWARDS SE $\left(: \mathrm{X}_{2}\right)$

$\left(: Y_{2}\right)$

PD

FD :MOD

MARCA-EXTREMO

PU

HOME

END

siendo MARCA-EXTREMO

TO MARCA-EXTREMO

RT 90

FD 3

BK 3

LT 90

END ${ }^{14}$

De esta forma los alumnos, después de haber representado un vector $\overrightarrow{\mathrm{AB}}$ a través del procedimiento REPRE-VECTORES y calculado su módulo $|\mathrm{AB}|$ mediante MODULO-VECTOR, pueden verificar su corrección. Si coinciden la marca (trazo perpendicular) indicadora del fin del segmento que representa el módulo con la flecha del vector representado, entonces tanto el cálculo realizado como los procedimientos desarrollados son correctos.

\section{Objetivo $b_{4}$}

Una de las estrategias que pueden seguir los alumnos para demostrar con el microordenador, utilizando el lenguaje LOGO, que la relación de equipolencia es una relación de equivalencia, es la siguiente ${ }^{15}$ :

- Propiedad reflexiva: A pesar de la evidencia de que cualquier vector $\overrightarrow{\mathrm{AB}}$ es equipolente consigo mismo, los alumnos pueden hacer la siguiente verificación gráfica:

- Punto medio $M$ de $\overrightarrow{A B}$

- Punto medio $M^{\prime}$ de $\overrightarrow{A B}$

- Como $M$ y $\mathrm{M}^{\prime}$ coinciden gráficamente, $\overrightarrow{\mathrm{AB}}$ es equipolente consigo mismo.

- Propiedad simétrica: $\mathrm{Si} \overrightarrow{\mathrm{AB}}$ es equipolente con $\overrightarrow{C D}$, entonces $\overrightarrow{C D}$ es equipolente con $\overrightarrow{\mathrm{AB}}$.

- Se calcula el punto medio de $\overline{\mathrm{AD}}$ y el punto medio de $\overrightarrow{\mathrm{BC}}$. Como $\overrightarrow{\mathrm{AB}}$ es equipolente con $\overrightarrow{\mathrm{CD}}$, ambos puntos medios coinciden.

- Se calcula el punto medio de $\overline{\mathrm{CB}}$ y de $\overline{\mathrm{DA}}$. Coincide gráficamente porque $\overline{\mathrm{CB}}$ $=\overline{\mathrm{BC}}$ y $\overline{\mathrm{DA}}=\widehat{\mathrm{AD}}$. Luego $\overline{\mathrm{CD}}$ es equipolente con $\overrightarrow{\mathrm{AB}}$.

- Propiedad transitiva: Si $\overrightarrow{\mathrm{AB}}$ es equipolente con $\overrightarrow{\mathrm{CD}}$ y $\overrightarrow{\mathrm{CD}}$ es equipolente con $\overrightarrow{\mathrm{EF}}$, entonces se debe cumplir que $\overrightarrow{\mathrm{AB}}$ es equipolente con $\mathrm{EF}$.

- Verificar gráficamente que $\overrightarrow{\mathrm{AB}}$ es equipolente con $\overrightarrow{\mathrm{CD}}$ y que $\overrightarrow{\mathrm{CD}}$ lo es con $\overrightarrow{\mathrm{EF}}$.

$\rightarrow$ Verificar con el microordenador que $\overrightarrow{\mathrm{AB}}$ es equipolente con $\overrightarrow{\mathrm{EF}}$. Para ello: Calcular gráficamente el punto medio de $\overline{\mathrm{AF}}$. - Calcular $g$ ráficamente el punto medio de $\overline{\mathrm{BE}}$.

- Observar la pantalla y comprobar si coinciden ambos puntos medios.

Entonces $\overrightarrow{\mathrm{AB}}$ será equipolente con $\overrightarrow{\mathrm{EF}}$.

Comprobadas estas tres propiedades, los alumnos habrán demostrado que la relación de equipolencia es una relación de equivalencia.

\section{Objetivo $b_{5}$}

Dado un vector $\overrightarrow{\mathrm{AB}}$ perteneciente a la clase $(\overrightarrow{\mathrm{AB}})$, representar un vector perteneciente a esa clase que tenga su origen en el punto $\mathrm{C}:(\mathrm{Xc}, \mathrm{Yc})$.

Los datos conocidos por los alumnos 
para desarrollar este objetivo serán las coordenadas de los dos puntos del vector dado $\mathrm{A}=(\mathrm{X}, \mathrm{Ya})$ y $\mathrm{B}=(\mathrm{Xb}, \mathrm{Yb})$ y las del punto inicial $\mathrm{C}=(\mathrm{Ca}, \mathrm{Cb})$ del que tienen que trazar.

Para representar este segundo vector necesitan conocer el punto final o el ángulo de inclinación respecto al eje de ordenadas o al de abscisas.

Dos de las posibles estrategias utilizadas por los alumnos para representar el vector $\overrightarrow{\mathrm{CD}}$ perteneciente a la clase $(\overrightarrow{\mathrm{AB}})$, son:

a) Calcular las coordenadas del punto $\mathrm{D}$, basándose en la relación que mantienen los puntos A y $\mathrm{B}$, para ello tendrán que:

- Hallar la diferencia de abscisas de los puntos inicial y final de los vectores de esa clase $\mathrm{Dx}=\mathrm{Xb}-\mathrm{Xa}$

- Hallar la diferencia en ordenadas $\mathrm{Dy}=\mathrm{Yb}-\mathrm{Ya}$

- Calcular el punto desconocido $\mathrm{Xd}=\mathrm{Xc}+\mathrm{Dx}$

$$
\mathrm{Yd}=\mathrm{Yc}+\mathrm{Dy}
$$

La validez de este razonamiento la pueden comprobar con el siguiente procedimiento

TO CONOCIMIENTO-PUNTO $: \mathrm{Xa}: \mathrm{Xb}: \mathrm{Xc}: \mathrm{Ya}: \mathrm{Yb}: \mathrm{Yc}$

(PRINT [LAS COORDENADAS DEL PUNTO D SON = ] :Xc + DIFERENCIA $(: X a-: X b): Y c+$ DIFERENCIA $\left.\left(: Y_{a}-: Y b\right)\right)$

\section{END}

Mediante el descubrimiento del ángulo de inclinación. Para ésto deberán:

b) Calcular el módulo del vector dado: $\overrightarrow{\mathrm{AB}}$.

- Descubrir el ángulo de inclinación $\hat{\mathrm{B}}$ que tiene el vector $\overrightarrow{\mathrm{AB}}$ respecto al eje de ordenadas.

- Ordenar à la "tortuga»: a) que se sitúe en $\mathrm{C}$; b) que gire a la derecha 8 grados; c) que avance el valor del módulo. $\mathrm{De}$ esta forma se representa el vector $\overrightarrow{\mathrm{CD}}$ equipolente con $\overrightarrow{\mathrm{AB}} y$, por lo tanto, perteneciente a la misma clase de equivalencia.

La cuestión está en cómo no conocer el ángulo de inclinación $\hat{\mathrm{B}}$.

Una forma de descubrirlo es: a) situar a la tortuga en el punto $\mathrm{A}:(\mathrm{Xa}, \mathrm{Xb}) \mathrm{mi}$ rando al Norte (orientación inicial); b) or- denarle que gire a la derecha un ángulo $\mathrm{B}$ cualquiera; c) indicarle que avance el valor del módulo; d) ordenarle que nos diga cuáles son sus coordenadas en ese punto.

Si las coordenadas que indica la tortuga corresponden con las del punto $\mathrm{B}$, quiere decir que el ángulo $\hat{B}$ girado es el que forma el vector $\overrightarrow{\mathrm{AB}}$ con el eje de ordenadas. $\mathrm{Si}$ no coinciden se varía el ángulo $\hat{\mathrm{B}}$, acotando el campo de búsqueda, hasta conseguirlo. El procedimiento utilizado para estos ensayos sería:

VALOR-ANGULO :B :L :Xa :Ya ${ }^{16}$ PU

SEPTOS SE (:Xa) (:Ya)

RT :B

$\mathrm{FD}: \mathrm{L}$

(PRINT XCOR YCOR)

PU

HOME

END

\section{Objetivo $b_{6}$}

Suma de los vectores libres: $(\overrightarrow{A B)},(\overrightarrow{B C})$.

- Los alumnos podrán conocer el módulo, la dirección y el sentido del vector suma resultante, de la siguiente forma:

- Dirección y sentido del vector suma: Podrán seguir las siguientes fases:

- Representar un vector $\overrightarrow{\mathrm{AB}}$ perteneciente a la clase $(\overrightarrow{\mathrm{AB}})(17)$. Para ello entre otras estrategias pueden utilizar el procedimiento: $: \mathrm{X}_{2}$

REPRE-VECTORES :N : $\mathrm{X}_{1}: \mathrm{Y}_{1}: \mathrm{X}_{2}$ - Trazar el vector $\overrightarrow{\mathrm{BC}}$, representante de la clase $(\overrightarrow{B C})$ que tenga su origen en el punto $\mathrm{B}=\left(\mathrm{X}_{2}, \mathrm{Y}_{2}\right)$ y extremo en $\mathrm{C}=\left(\mathrm{X}_{3}\right.$, $\mathrm{Y}_{3}$ )

- También a través del procedimiento REPRE-VECTORES :N : $\mathrm{X}_{1}: \mathrm{Y}_{1}: \mathrm{X}_{3}: \mathrm{Y}_{3}$

Se representará el vector $\overrightarrow{A C}$ dado por el punto $A=\left(X_{1}, Y_{1}\right) ; C=\left(X_{3}, Y_{3}\right)$

De esta forma conocen la dirección y el sentido.

- Módulo del vector suma.

- El módulo del vector resultante de la operación suma lo pueden conocer los alumnos utilizando el procedimiento MODULO-VECTOR; con los siguientes parámetros de entrada:

$: N: X_{1}: Y_{1}: X_{3}: Y_{3}$

Queremos recordar en este punto que una de las ventajas que ofrece el microor- 
denador es la posibilidad que tiene de pro-porcionar refuerzos gráficos de aprendiza$\mathrm{je}$ a los alumnos.

Aplicando esta posibilidad al objetivo que acabamos de desarrollar, los alumnos después de haber representado y calculado el módulo del vector suma $\widehat{A C}$, pueden utilizar, como se hizo en el objetivo $b_{3}$ ), el procedimiento VERIFICACIONVALOR-MODULO para comprobar la corrección del valor hallado.

\section{Objetivo $b_{7}$}

Definir correctamente las propiedades conmutativa, asociativa, elemento neutro y simétrico de la suma de dos vectores libres:

\section{a) Propiedad conmutativa}

Los alumnos utilizando los procedimientos precedentes pueden demostrar que:

$10^{\circ} \overrightarrow{\mathrm{AB}} \sim \overrightarrow{\mathrm{DC}}$ ambos pertenecientes a la clase $[\overrightarrow{A B}]=a$; y que

$\overrightarrow{\mathrm{AD}} \sim \overrightarrow{\mathrm{BC}}$ ambos pertenecientes a la clase $[\mathrm{BC}]=\mathrm{b}$.

$2^{\circ} \overrightarrow{\mathrm{AB}}+\dot{\mathrm{BC}}=\overrightarrow{\mathrm{AC}} \in \mathrm{a}+\mathrm{b}, \mathrm{y} \overrightarrow{\mathrm{AD}}+$ $\overrightarrow{\mathrm{DC}}=\overline{\mathrm{AC}} \in \mathrm{b}+\mathrm{a}$.

$3 .^{\circ}$ De lo anterior se deduce que a + $b=b+a$.

Estas propiedades pueden observarse en la figura 3:

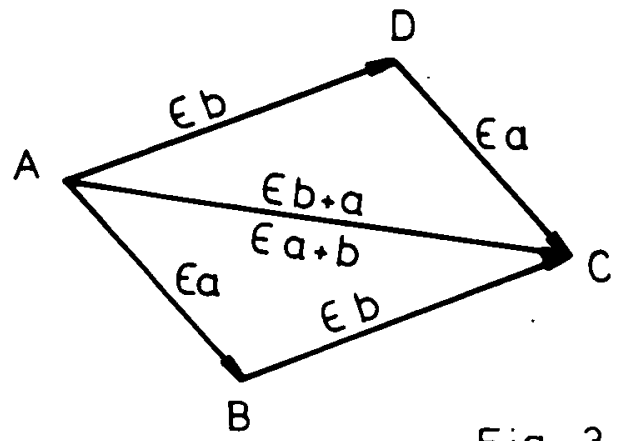

Fig. 3

\section{b) Propiedad asociativa}

Los alumnos de igual forma podrán demostrar que:

1. $\overrightarrow{A C} \in \mid a+b, \overrightarrow{B D} \in b+c$. 2. $\overrightarrow{\mathrm{AD}} \in \mid(\mathrm{a}+\mathrm{b})+\mathrm{c}$.
3. $\overrightarrow{\mathrm{AD}} \in \mathrm{a}+(\mathrm{b}+\mathrm{c})$.

4. De lo anterior se deduce que $(a+$

b) $+c=a+(b+c)$.

Como se expresa en la figura 4 :

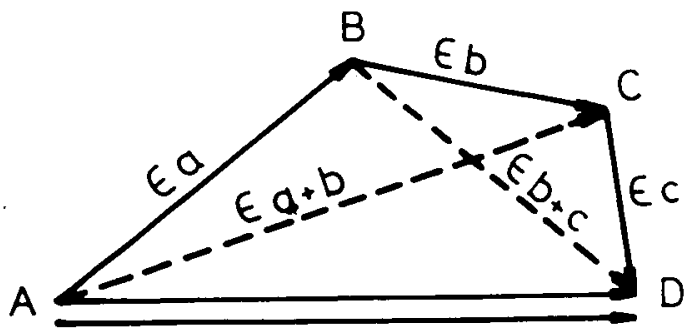

Fig. 4

\section{c) Elemento neutro}

De forma análoga los alumnos podrán definir que para todo vector libre $[\overline{\mathrm{AB}}]=$ a.

$$
a+0=0+a=a .
$$

\section{d) Elemento simétrico}

Los alumnos pueden verificar que para cualquier vector libre $[\overrightarrow{\mathrm{AB}}]=$ a existe otro $[\overrightarrow{\mathrm{BA}}]=-a$, tal que $a+(-a)=0$ (Fig. 5 ).

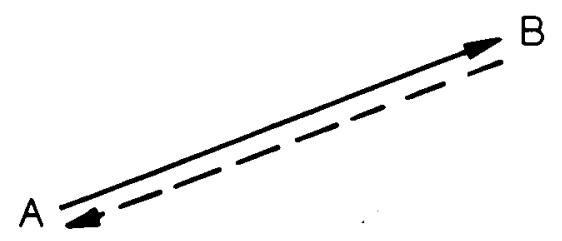

Fig. 5

Antes de pasar al objetivo $b_{8}$ el profesor hará ver a los alumnos que por estas propiedades el conjunto de todos los vectores libres del plano forman, respecto de la suma, un grupo abeliano denominado GRUPO ADITIVO DE LOS VECTORES DEL PLANO.

Objetivo $b_{8}$

Calcular y representar la diferencia de los vectores libres (Fig. 6). 


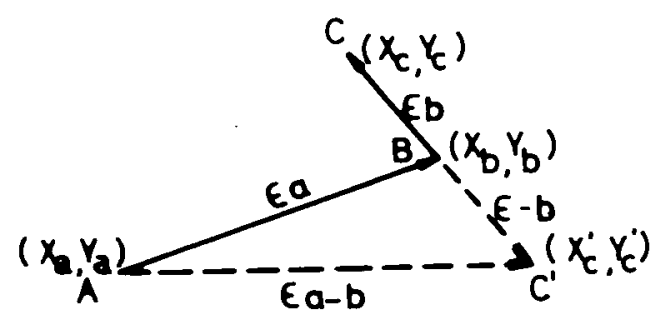

Fig. 6

Como $\mathrm{a}-\mathrm{b}=\mathrm{a}+(-\mathrm{b})$ los alumnos deberán conocer las coordenadas $\mathrm{X}_{\mathrm{c}} \mathrm{Y}^{\prime}{ }_{\mathrm{c}}$ $\mathrm{de}-\mathrm{b}$ para poder posteriormente representar y calcular el módulo del vector $\mathrm{AC}^{\prime}$ $\in \mathbf{a}-\mathbf{b}$.

Uno de los procedimientos que los alumnos pueden utilizar es:

TO COORDENADAS-VECTOR-OPUESTO :MOD $: \mathrm{X}_{\mathrm{b}}: \mathrm{Y}_{\mathrm{b}}: \mathrm{X}_{\mathrm{c}}: \mathrm{Y}_{\mathrm{c}}$ PU

SEPTOS SE $\left(: \mathrm{X}_{\mathrm{b}}\right)\left(: \mathrm{Y}_{\mathrm{b}}\right)$ $\left(: Y_{C}\right)$

SETHEADING TOWARDS SE $\left(: X_{c}\right)$

RT 180

FD : MOD

FLECHA

(PRINT [LAS COORDENADAS DEL VECTOR-BC SON $\left.\mathrm{X}_{\mathrm{B}}=\right]: \mathrm{X}_{\mathrm{h}}$ $\left[\mathrm{Y}_{\mathrm{B}}=\right]: \mathrm{Yc}_{\mathrm{c}}\left[\mathrm{X}_{\mathrm{c}}^{\prime}=\right] \mathrm{XCOR}\left[\mathrm{Y}_{\mathrm{c}}^{\prime}=\right]$ YCOR)

PU

HOME

END $^{18}$

Conocidas estas coordenadas los alumnos, utilizando el procedimiento REPREVECTORES, pueden representar el vector $\overrightarrow{\mathrm{AB}}$, el $-\overrightarrow{\mathrm{BC}}$ y el resultante de la diferencia $-\overrightarrow{A C}$.

Objetivo b, Cálculo y representación del resultado de la multiplicación de un vector libre por un número real

El alumno puede conseguir este objetivo utilizando varias estrategias.

Dos de ellas son:

a) Dados el valor de un número real $\mathrm{R}$ y las coordenadas de los puntos inicial $y$ final A: $\left(X_{1}, Y_{1}\right)$, B: $\left(X_{2}, Y_{2}\right)$ de un yector $\overrightarrow{\mathrm{AB}}$ perteneciente a la clase $[\overrightarrow{\mathrm{AB}}]$, el alumno podrá:
- Calcular el módulo del vector $\overrightarrow{\mathrm{AB}}$ mediante el procedimiento MODULO-VECTOR.

- Obtener otro $\overrightarrow{\mathrm{AB}}$ de la misma dirección y sentido y de módulo el producto del módulo de $\overrightarrow{\mathrm{AB}}$ por el número real dado.

b) Con las mismas condiciones, el alumno podrá:

- Multiplicar las diferencias de coordenadas $X_{2}-X_{1}, Y_{2}-Y_{2}$ por el número real.

- Los valores obtenidos corresponderán a las coordenadas finales del nuevo vector producto.

- Representar el nuevo vector.

Esta segunda forma de resolución la puede hacer el alumno con el siguiente procedimiento:

TO PROD-VECTOR-POR-NUM. $R E A L: R: X_{1}: Y_{1}: X_{2}: Y_{2}$

PU

SETPOS SE $\left(: X_{1}\right)\left(: Y_{1}\right)$

SETHEADING TOWARDS SE $\left(: \mathrm{X}_{2}\right)$ $\left(: Y_{2}\right)$

PD

SETPOS SE (PRODUCT1 :R : $\mathrm{X}_{1}: \mathrm{X}_{2}$ ) (PRODUCT2 :R : $Y_{1}: Y_{2}$ )

FLECHA

PU

HOME

END

Siendo los procedimientos PRODUCT 1 Y PRODUCT 2.

TO PRODUCT $1: \mathrm{R}: \mathrm{X}_{1}: \mathrm{X}_{2}$ OUTPUT $\left(: \mathrm{X}_{2}-: \mathrm{X}_{1}\right) *: \mathrm{R}$ END

TO PRODUCT $2: R: Y_{1}: Y_{2}$ OUTPUT $\left(: Y_{2}-: Y_{1}\right) *: R$ END

Donde $\mathrm{R}$, es el valor del número real; $\left(\mathrm{X}_{1}, \mathrm{Y}_{1}\right),\left(\mathrm{X}_{2}, \mathrm{Y}_{2}\right)$ las coordenadas respectivas de los puntos $\mathrm{A}$ y $\mathrm{B}$ del vector $\overrightarrow{\mathrm{AB}}$ perteneciente a la clase $[\overrightarrow{\mathrm{AB}}]$.

\section{Objetivo $b_{10}$}

Definir correctamente las propiedades $r$ $(a+b)=r a+r b ;(r+s) a=r a+s a ;$ $(r s) a=r(s a) ; l a=a$ siendo $a$ y $b$ vectores libres y $r, s$ números reales. 
Feld Dados los vectores $\overrightarrow{\mathrm{AB}} \in$ : a y $\overrightarrow{\mathrm{BC}} \in \mathrm{b}$ los estudiantes mediante los procedimientos creados para el desarrollo de los objetivos anteriores están en condiciones para demostrar las siguientes propiedades:

-a) $r(a+b)=r a+r b$ (Fig. 7).

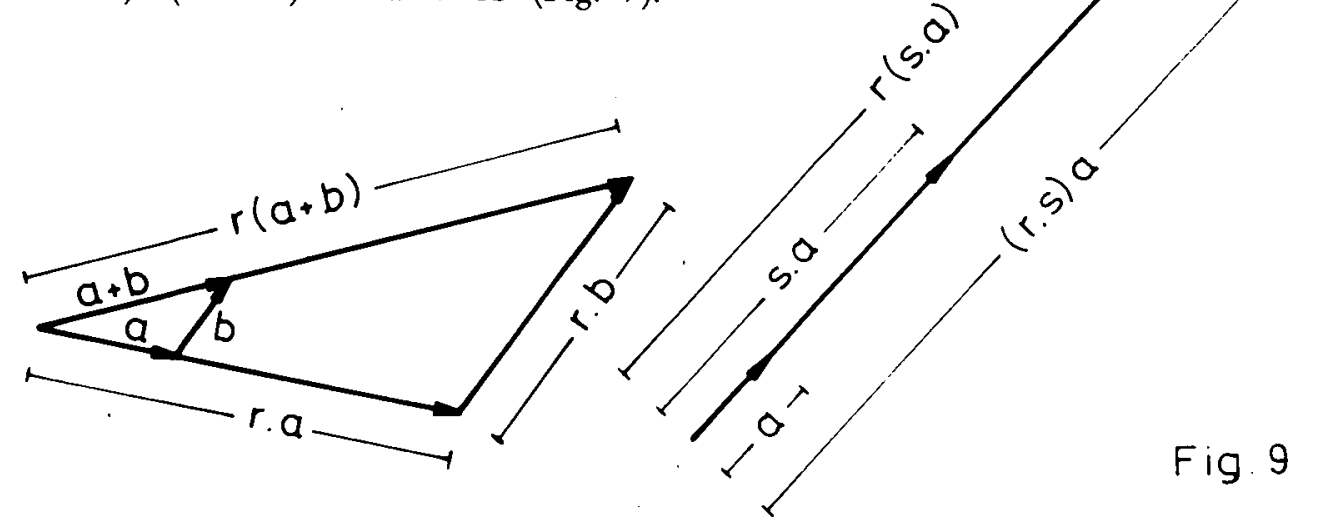

Fig. 7

$\left.a_{1}\right)$ Calcular y representar $a+b^{19}$.

$\left.a_{2}\right)$ Calcular y representar $\mathrm{r}(\mathrm{a}+\mathrm{b})$ mediante el procedimiento PRODUCTVECTOR-POR-NUM-REAL.

$a_{3}$ ) Con el mismo procedimiento calcular y representar ra y $\mathrm{rb}$. rb.

$a_{4}$ ) Comprobar que $r(a+\cdot b)=r a+$ -b) $(r+s) a=r a+s a$ (Fig. 8).

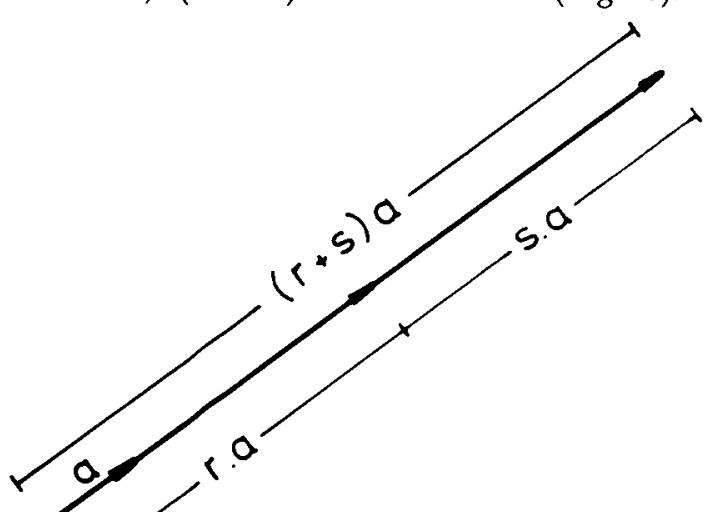

Fig. 8

Esta propiedad la pueden demostrar los alumnos siguiendo la misma estrategia que en la anterior y utilizando el procedimient o: PROD.VECTOR - POR NUM'REAL.

$$
-c) \quad(r s) a=r(s a) \text { (Fig. 9). }
$$

Dados dos números reales $r$ y $s$ y las coordenadas iniciales $\left(\mathrm{X}_{1}, \mathrm{Y}_{1}\right)$ y finales $\left(\mathrm{X}_{2}, \mathrm{Y}_{2}\right)$ de un vector $\overrightarrow{\mathrm{AB}} \in$ a, los alumnos podrán: $\left.c_{1}\right)$ Calcular y representar $\left(r^{*} s\right)$ a, utilizando el procedimiento PROD'VECTOR-POR-NUM'REAL.

El valor del número real a introducir en el procedimiento será el resultado del producto $r$ 's.

$c_{2}$ ) Utilizar dos veces consecutivas el procedimiento anterior. Una para el valor del número real s. Otra para multiplicar el módulo del vector resultante por el número real $r$.

$\left.c_{3}\right)$ Verificar que $(r s) a=r(s a)$.

-d) la = a. Esta propiedad la pueden demostrar los alumnos con estrategias y procedimientos LOGO análogos a los anteriores. La única variante se centra en que el número real por el que se multiplica el vector es 1 .

\section{Objetivo $b_{11}$}

Dados dos vectores libres $a_{1}$ y $a_{2}$, dibujar un representante de la combinación lineal $r^{\prime} a_{1}+s^{\prime} a_{2}$ (Fig. 10).

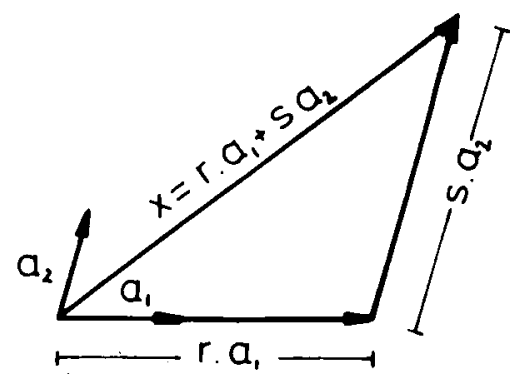

Fig. 10 
Siendo $\overrightarrow{A B} \in a_{1}$ y $\overrightarrow{C D} \in a_{2}$ dos vectores lineales independientes del plano, cuyas coordenadas respectivas son $\mathrm{A}$ : (Xa, Ya) B: (Xb, Yb), C: (Xc, Yc), D: (Xd, Yd), los alumnos pueden calcular y representar cualquier otro vector del plano como resultado de la suma de múltiplos escalares de ambos (combinación lineal). Para ello los estudiantes.

- Elegirán un vector $\overrightarrow{\mathrm{AB}} \in \mathrm{a}_{1}$. Lo representarán y calcularán su módulo utilizando los procedimientos REPRE-VECTORES Y MODULOVECTOR.

- Calcularán y representarán el vector resultante de multiplicar $\overrightarrow{\mathrm{AB}}$ por un número real $\mathrm{r}$ mediante el procedimiento PRO-VECTOR-PORNUM-REAL.

- Representarán un vector $\overrightarrow{\mathrm{CD}} \in \mathrm{a}_{2}$ que tenga su origen de coordenadas en el punto $\mathrm{B}:(\mathrm{Xb}, \mathrm{Yb})$ tal como se hizo en el desarrollo del objetivo $b_{5}$.

- Calcularán y representarán el vector resultante de multiplicar el vector $\overrightarrow{C D}$ por el número real $s$.

Siguiendo los pasos apuntados para el objetivo $b_{6}$, se puede hallar y representar el vector que surja de la suma de ambos, que será combinación lineal de ellos: $\mathrm{X}=$ $r a_{1}+s a_{2}$.

\section{PLANIFICACION DE CONTENIDOS}

Como se ha venido especificando, al desarrollar los objetivos, para poder descubrir, investigar y en definitiva aprender los contenidos sobre espacios vectoriales con microordenador, era necesario que los estudiantes, previamente para cada objetivo, conocieran correctamente una serie de órdenes del lenguaje LOGO.

Un esquema de la secuencia seguida por los alumnos en el aprendizaje de los dos tipos de contenidos, es el siguiente: ${ }^{20}$

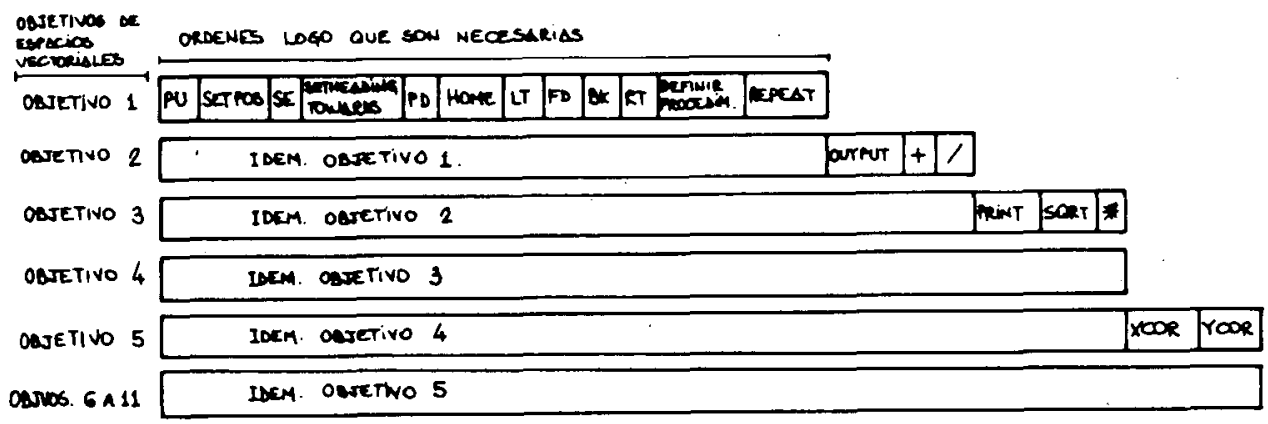

Resumiendo este esquema en una representacion lineal,quedaria asi: (20)

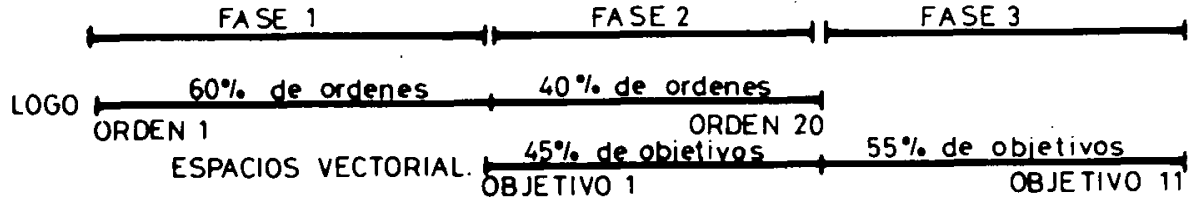

Las fases resultantes corresponden a:

FASE 1: Aprendizaje de contenidos auxiliares sobre lenguaje LOGO.

FASE 2: Aprendizaje conjunto de ambos tipos de contenidos. Además de introducir órdenes nuevas sobre LOGO, los alumnos utilizan las conocidas en la fase 1.

FASE 3: Aprendizaje de contenidos sobre espacios vectoriales. No se introduce ninguna orden LOGO nueva, utilizan las conocidas en fases anteriores. 
Además de la prioridad en el tiempo de las órdenes LOGO como base para la adquisición de los objetivos sobre espacios vectoriales, otras razones por las que hemos realizado esta planificación se refieren:

a) A la conveniencia de evitar una interferencia prolongada en la presentación a los alumnos de conceptos nuevos de ambos tipos de contenidos.

b) Que permite a los estudiantes durante un período de tiempo (fase 3) aplicar todos los contenidos auxiliares introducidos en esta unidad para adquirir una parte de los objetivos de la otra materia del currículo.

\section{CONCLUSIONES}

La exposición de los apartados anteriores nos permite hacer las siguientes consideraciones finales:

- Es posible planificar conjuntamente unos contenidos informáticos con los de otras áreas de conocimiento. Queda un gran campo de investigación abierto para conocer si este tipo de organización es más efectivo que otros para conseguir que los alumnos comprendan y apliquen mejor y con mayor rapidez estos contenidos. También desde un punto de vista de la Psicología del Pensamiento es necesario saber qué tipo de planificación desarrolla más eficazmente estrategias de resolución de problemas en los estudiantes.

- Respecto a la consideración procedente, en el desarrollo de los objetivos sobre espacios vectoriales, hemos ido constatando cómo el uso del microordenador, utilizando el lenguaje de programación LOGO, amplía el número de estrategias que pueden utilizar los alumnos para resolver problemas y favorecer la ejecución de las mismas.

- Desde un punto de vista del aprendizaje de unos contenidos sobre espacios vectoriales y a través de la propuesta metodológica que hemos efectuado observamos que el lenguaje LOGO, basado en la geometría de la «tortuga», permite representar los vectores de forma diferente a como lo hace la geometría del «papel, la regla y el lápiz».

Este hecho permite a los alumnos, durante el proceso de investigación para conseguir los objetivos, comparar ambas representaciones y trasladar las descripciones de una representación dentro de la otra. Estos cambios de descripción producen un conjunto de estímulos y refuerzos que no aparecerían en el caso de una sola representación.

- Por último, respecto a la planificación de ambos tipos de contenidos consideramos que no deben introducirse simultáneamente durante todo el proceso de aprendizaje. Esto llevó a proponer tres fases escalonadas:

a) La primera se dedicaba exclusivamente a la introducción de contenidos informáticos.

b) En la segunda se solapan ambos tipos de contenidos.

c) En la tercera sólo se introducen objetivos del área de conocimiento (en nuestro caso los espacios vectoriales). En esta fase se sigue aplicando los contenidos informáticos introducidos en las anteriores. 
${ }^{1}$ Denominamos a los contenidos informáticos «auxiliares", no porque tengan menos importancia o sean menos fundamentales, sino porque en esta organización de materias del currículo, el conocimiento de los primeros van a permitir al alumno comunicarse con el microordenador para comprender los segundos.

2 Siguiendo a Fultrell, M. K. and Geiser, P. (1984); Bunderson, C. V (1984) y Criswell, E. L. y Swezey, R. W (1984) el término "Courseware», hace referencia a los programas de ordenador que tienen fines instructivos.

${ }^{3}$ Las órdenes que señalamos están en inglés porque el intérprete LOGO que hemos utilizado para construir y verificar los programas que exponemos a continuación, es el LCS para APPLE que viene en inglés. Siendo conscientes de la dificultad que esto supone para los alumnos, proponemos como actividad al profesor interesado que traduzcan estas instrucciones utilizando procedimientos. Por ejemplo para traducir la instrucción:

FORDWARD X por AVANZA X sería:

TO AV :X

FD :X

\section{END.}

(Todos estos procedimientos se salvarán con el comando SAVE antes de apagar el equipo. Al reanudar la actividad se recuperan con LOAD.)

${ }^{4}$ Gimeno Sacristán, J. "Teoria de la enseñanza y desarrollo del currículo», p. 207.

${ }^{5}$ Pérez Gómez, A. I. "Aprendizaje, desarrollo y enseñanza", p. 22.

${ }^{6}$ Una situación problemática se diferencia, entre otros aspectos, de los ejercicios, prácticas, problemas de repetición de algún algoritmo previamente enseñado por el profesor, en que están elaboradas partiendo de las necesidades e intereses de los alumnos a los que van dirigidas y que en su enunciado se presentan algunas informaciones sobre los contenidos objeto de aprendizaje.

7 Respecto al esquema de planificación de la secuencia de objetivos sobre lenguaje de programación LOGO que deben conocer los alumnos de forma previa, como auxiliares para adquirir los objetivos de espacios vectoriales, se expone al final del artículo, en el apartado "Planificación de contenidos".

${ }_{B}^{B}$ Watt (1984), Reggini (1982) y Papert (1980), apuntan que, los errores cometidos son fuentes de aprendizaje, por el reto motivante que suponen al alumno volver a revisar el programa, descubrir el error, corregirlo, ejecutarlo y volver a comprobar la exactitud de los resultados obtenidos.

${ }^{9}$ Según sea un compilador o intérprete el conjunto de programas utilizados para programar al microordenador con el lenguaje LOGO.

${ }^{1} O$ Los objetivos sobre lenguaje LOGO los conseguirán los alumnos mediante la explicación del profesor, la comprobación en el microordenador y utilizándolos como medio para conseguir los objetivos sobre espacios vectoriales.

${ }^{1} 1$ Puede observarse que el procedimiento REPRE-VECTORES tiene cinco parámetros de entrada, $N$ indica el número o letra que identifica el vector que se va a representar. $X_{1}, Y_{1}$ las coordenadas del punto inicial. $\mathrm{X}_{2}, \mathrm{Y}_{2}$ las del punto final del vector. La orden PU hace que la "tortuga" levante la pluma y no deje rastro cuando se sitúe, al ejecutar la orden SEPTOS SE $\left(: X_{1}\right)\left(: Y_{1}\right)$, en el punto $X_{1} Y_{1}$.

SETHEADING TOWARDS SE $\left(: \mathrm{X}_{2}\right)\left(: Y_{2}\right)$ gira la «cabeza" de la tortuga hacia el punto $\left(\mathrm{X}_{2} \mathrm{Y}_{2}\right)$. La orden PD baja la pluma de la tortuga para que deje rastro cuando vaya al punto $\mathrm{X}_{2} \mathrm{Y}_{2}$ al ejecutar la orden SEPTOS SE $\left(: \mathrm{X}_{2}\right)\left(: \mathrm{Y}_{2}\right)$. PU vuelve a levantar la pluma para que no deje rastro al volver la tortuga a casa.

${ }^{1} 2$ Esta situación problemática puede ser del siguiente tipo: (los contenidos están presentes en el enunciado del problema).

«Si dos vectores $\overrightarrow{\mathrm{AB}}$ y $\overrightarrow{\mathrm{CD}}$ de un plano, son equipolentes cuando los puntos medios de $\overrightarrow{\mathrm{AD}}$ y $\overrightarrow{\mathrm{CD}}$ coinciden, ¿son ęquipolentes las fuerzas que realizan dos amigos; representadas por los vectores $\overrightarrow{A B}:(-40,20)$, $(50,80)$ y $\overline{C B}:(30,-25),(120,35)$ para subir una maleta a la baca de un coche?

${ }_{3} 3$ Denominamos $\left(\mathrm{X}_{1}, \mathrm{Y}_{1}\right)\left(\mathrm{X}_{2}, \mathrm{Y}_{2}\right)$ las coordenadas del primer vector $\mathrm{y}\left(\mathrm{X}_{3}, \mathrm{Y}_{3}\right),\left(\mathrm{X}_{4}, \mathrm{Y}_{4}\right)$ las del segundo.

${ }_{4} 4$ Para trazar la longitud que representa el valor del módulo se ha utilizado la orden FD: Módulo, (la tortuga avanza el valor del módulo). Para señalar el extremo de ese segmento se ha utilizado el procedimiento MARCA-EXTREMO.

${ }_{15} 5$ Los procedimientos LOGO a utilizar en cada uno de los pasos siguientes, correspondientes a la demostración de las tres propiedades que debe cumplir la relación equipolencia para que sea de equivalencia, son los mismos que se han expuesto anteriormente para verificar gráficamente si son o no equipolentes los vectores.

16 Los alumnos previamente deben conocer las órdenes LOGO: XCOR, YCOR.

La entrada: $B$ representa el ángulo de giro que se ensaya. $: \mathrm{L}$ el valor del módulo del vector $\overrightarrow{\mathrm{AB}}$. $: \mathrm{Xa}$, $: Y b$ las coordenadas del punto inicial $A$ de ese vector.

$17 \mathrm{Si}$ un alumno en yez del vector $\overrightarrow{\mathrm{AB}}$ elige otro representante $\overrightarrow{\mathrm{A}^{\prime}} \mathrm{B}^{\prime}$, de la clase $(\overrightarrow{\mathrm{AB}})$ al final del proceso llegará a un vector $\overrightarrow{A^{\prime} C}$ ' equipolente con $\overrightarrow{A C}$ y por eso perteneciente a la misma clase $(\overrightarrow{A C})$.

${ }_{1} 8 \mathrm{En}$ la pantalla aparece el siguiente texto, indicando las coordenadas iniciales y finales del vector diferencia:

LAS COORDENADAS DEL VECTOR-BC SON XB $=\mathrm{YB}=\mathrm{X}^{\prime} \mathrm{C}=\mathrm{Y}^{\prime} \mathrm{C}=$

19 Realizado en el objetivo $b_{6}$.

20 Para mostrar la idea de la planificación seguida en el siguiente esquema se ha calculado y representado los porcentajes de órdenes del lenguaje LOGO y de los objetivos de espacios vectoriales. 
Este articulo presenta la posibilidad de programar conjuntamente unos contenidos informáticos con los d e otras materias del curriculo escolar. Después de ilustrar esta posibilidad mediante la programación de unos contenidos sobre espacios vectoriales y lenguaje LOGO de programación de ordenadores, se indican algunos aspectos positivos que favorecen los procesos de aprendizaje de los alumnos.

\section{Abstract}

This paper shows bow to jointly program computer-related subjects together with otber school curriculum subjects. Following the illustration of this technique applied to the programming of materials about vector spaces and LOGO computer language, we indicate some positive aspects of our technique that make the learning proceses easier for the students.

\section{Résumé}

Cet article présente la possibilité de programmer ensemble contenues informatiques avec autres matiéres du curriculum scolaire. Apres d'illustrer cette possibilité avec la programmation de quelques contenus sur espaces vecteurs et language LOGO pour la programmation d'ordinateurs, nous signalons des aspects positifs qui favorisent les mecanismes d'apprentissage des èléves.

\section{Referencias}

Abelson, H. y DisessA, A.: Turtle Geometry. The MIT Press. Massachusetts, 1984.

BILISTEIN, R.: "The Role of Technology in Mathematics Education: Wats Your View. The Computing Teacher. April, pp. 19-20, 1985.

Bunderson, C. V.: "Courseware", in H. F. O'Neill, Jr. (Ed.), Computer-Based Instruction: A State-of-theArt Assessments. New York. Academic. Press., 1981.

BuTrs, Th.: "Posing Problems Properly", in N.C.T.M. Problem Solving in School Mathematics. Yearbook. Reston. Virginia, pp. 23-33, 1980.

CATHEART, W. G.: "Problem solving using the microcomputer", in IFP. Informatics in elementary education. J. Tindsley y E. B. Tagg. Ed. Noth Holland, pp. 115-118, 1984.

CRISWELL, E. L. y SwEZEY, R. W.: «Behavioral Learning Theory-Based Computer Courseware Evaluation». Educational Technology. November, pp. 43-46, 1984.

CHOQUET, G.: "Introducción al libro de la Enseñanza de la Geometría", en J. Piaget y otros, La enseñanza de las matemáticas moáernas. Alianza Universidad. Madrid, 1983.

DE Lorenzo, J.: "La muerte de la Geometría". Revista de Bachillerato. Sup. n. 13 . Enero-marzo, pp. 31-34, 1980.

DEWEY, J.: How we think D. C. Heat and Company. Boston, pp. 106-115, 1983.

Dijkstra, E. W.: $A$ short introduction to the art of programming, Tech. Univ. Eindhoven, 1971.

Dromey, R. G.: How to Solve it by Computer, Prentice-Hall International Englewood Cliffs. N. J., 1982.

Fultreli, M. K. and Geiser, P.:The Well-Trained Computer. Englewood Cliffs, New Jersey, 1984.

GIMENO SACRISTAN, J.: Teoria de la enseñanza y desarrollo del curriculo. Anaya. Madrid.

HAYES, J. R.: Cognitive Psychology. Thinking and Creating. The Dorsey Press. Illinois, 1978.

Howe, J. et al: "Model Building, Matematics and LOGO", en Yazdani. M. Ed. New Horizons in Educational Computing. Ellis Horwood Lim. Chichester. Great Britain.

JaCOBson, M. H., LeSTER, F. K. y STENGER, A.: «Making Problem Solving Come Alive in the Intermediate Grades" in N.C.T.M. Problem Solving in School Mathematics, Yearbook, Reston, Virginia, pp. 127-135, 1980.

KLASSEN, D.: "Computer Literary". Topics January, 1981.

LANDA, L. N.: Algoritmos para la enseñanza y el aprendizaje. Trillas. México, pp. 11-12, 1978.

LAwLER, S.: "Designing Computer-Based Microworlds" in Yazdani M. Ed. New Horizons in Educational Computing. Ellis Horwood Lim. Chickester. Great Britain, 1984.

Malone, Th. W.: "Toward a Theory of Intrinsically Motivating Instruction" Cognitive Sciencie, n. ${ }^{\circ} 4, \mathrm{pp}$. 333-369, $1981 \mathrm{a}$.

Malone, Th. W.: “What Makes Computer Games Fun?" BYTE. Diciembre, pp. 258-275, 1981 .

MOLNAR, A.: "The next great crisis in American Education: Computer Literacy", Journal of Educational Tecbnology System, 1981.

OSBORNE, A., y KASTEN, M. B.: *Opinions about Problem Solving in the Curriculum for the 1980s: A Report*, en NCTM. Problem Solving in School Mathematics. Yearbook. Reston. VA, 1980.

PAPERT, S.: Mindstorms: Children, Computers and Powerful Ideas, Basic Books Inc. New York, 1980. 
PARADELA, L. F.: *Aspectos fundamentales del proyecto Atenea". Cuademos de Educación y Nuevas Tecnologías, núm. 2, pp. 11-14, 1985.

Perez Gómez, A. I.: «Aprendizaje, desarrollo y enseñanza» en Pérez Gómez, A. y J. Almaraz, Lecturas de aprendizaje y enseñanza. (Ed.) Zero Zyx. Madrid, pp. 11-31, 1981.

POLYA, G.: How to Solve it Princeton University. Precs. USA. Versión castellana, Como plantear y resolver problemas (Ed.:) Trillas, México, 1945.

REGGINI, H.: Alas para la mente (Ed.:) Galápago. B. Aires, 1982.

SSANTALO, L. A.: *Situación de la enseñanza de la Geometría frente a las nuevas tendencias de la educación matemática», Revista de Bachillerato. Sup. núm. 13, enero-marzo, pp. 23-29, 1980.

SHERARD III, W. H.: *Why is geometry a basic skill?», Mathematics Teacher. Jaunary, pp. 19-21, 1981.

SOLOMON, C.: \&Introducing LOGO to children*, Byte, 7, núm. 8, pp. 196-208, 1982.

TORGERSON, S.: Logo in the Classroom, ICCE Publications, pp. 107-109, 1984.

UNESCO: «New Frends in Mathematics Teaching». Vol. III, París, 1973.

WATT, D.: "Computer Literacy: What Shonuld School be doing". Classroom Computer News, 1 (2), pp. 26-27, 1980.

WATT, D.: Aprendiendo con LOGO, McGraw Hill. Madrid, 1984. 